\title{
A reply to Wiwanitkit
}

The quote in Prof. Wiwanitkit's letter is from the abstract rather than from the full text paper. The full text paper does deal with the enhancement of antigen retrieval, using both microwaving and enzyme (trypsin) treatment of the sections. In addition, steps to block endogenous peroxidase activity prior to incubation with the primary antibody are elucidated.

I believe this is what is meant by 'specimen preparation (preanalytical phase)'. There is also a clear description of measures taken to ensure specificity of the immunoperoxidase staining reaction. But the comments are well taken and due attention will, of course, be paid to the points raised when the test is developed for routine testing of samples.

\section{S Njiro}

Pathology Division, Onderstepoort Veterinary Institute, Private Bag X05, Onderstepoort, 0110 South Africa

Email: njiros@arc.agric.za 